\title{
Chromosome Isolation by Flow Sorting in Aegilops umbellulata and Ae. comosa and Their Allotetraploid Hybrids Ae. biuncialis and Ae. geniculata
}

\author{
István Molnár ${ }^{1}$, Marie Kubaláková ${ }^{2}$, Hana Šimková ${ }^{2}$, András Cseh ${ }^{1}$, Márta Molnár-Láng ${ }^{1}$, Jaroslav \\ Doležel $^{2 *}$
}

1 Agricultural Research Institute of the Hungarian Academy of Sciences, Martonvásár, Hungary, 2 Centre of the Region Haná for Biotechnological and Agricultural Research, Institute of Experimental Botany, Olomouc, Czech Republic

\begin{abstract}
This study evaluates the potential of flow cytometry for chromosome sorting in two wild diploid wheats Aegilops umbellulata and Ae. comosa and their natural allotetraploid hybrids Ae. biuncialis and Ae. geniculata. Flow karyotypes obtained after the analysis of DAPI-stained chromosomes were characterized and content of chromosome peaks was determined. Peaks of chromosome $1 \mathrm{U}$ could be discriminated in flow karyotypes of Ae. umbellulata and Ae. biuncialis and the chromosome could be sorted with purities exceeding $95 \%$. The remaining chromosomes formed composite peaks and could be sorted in groups of two to four. Twenty four wheat SSR markers were tested for their position on chromosomes of Ae. umbellulata and Ae. comosa using PCR on DNA amplified from flow-sorted chromosomes and genomic DNA of wheatAe. geniculata addition lines, respectively. Six SSR markers were located on particular Aegilops chromosomes using sorted chromosomes, thus confirming the usefulness of this approach for physical mapping. The SSR markers are suitable for marker assisted selection of wheat-Aegilops introgression lines. The results obtained in this work provide new opportunities for dissecting genomes of wild relatives of wheat with the aim to assist in alien gene transfer and discovery of novel genes for wheat improvement.
\end{abstract}

Citation: Molnár I, Kubaláková M, Šimková H, Cseh A, Molnár-Láng M, et al. (2011) Chromosome Isolation by Flow Sorting in Aegilops umbellulata and Ae. comosa and Their Allotetraploid Hybrids Ae. biuncialis and Ae. geniculata. PLoS ONE 6(11): e27708. doi:10.1371/journal.pone.0027708

Editor: Klaus F. X. Mayer, Helmholtz Center Munich, Germany

Received July 16, 2011; Accepted October 22, 2011; Published November 23, 2011

Copyright: (C) 2011 Molnár et al. This is an open-access article distributed under the terms of the Creative Commons Attribution License, which permits unrestricted use, distribution, and reproduction in any medium, provided the original author and source are credited.

Funding: This research was supported by the Hungarian National Research Fund (PD83444), Czech Ministry of Education, Youth and Sports (grant awards LC06004 and OC08025), and the European Regional Development Fund (Operational Programme Research and Development for Innovations No. CZ.1.05/2.1.00/ 01.0007). The funders had no role in study design, data collection and analysis, decision to publish, or preparation of the manuscript.

Competing Interests: The authors have declared that no competing interests exist.

*E-mail: dolezel@ueb.cas.cz

\section{Introduction}

Bread wheat (Triticum aestivum L.) is a natural allohexaploid $(2 \mathrm{n}=6 \mathrm{x}=42$, AABBDD), which evolved via two rounds of hybridization and polyploidization, involving several species of the Triticum and Aegilops genera [1]. The gene pool of elite hexaploid wheat is relatively narrow due to thousands of yearslong cultivation and domestication [2], undermining the ability to sustain the crop yield and quality under extreme environmental and biotic conditions. The remarkable diversity of related wild species offers a reservoir of novel alleles, favourable genes and gene complexes for wheat breeding by means of interspecific hybridization or transgene technology.

The genus Aegilops, which is the most closely related taxon to Triticum, contains eleven diploid and twelve polyploid species [3]. Seven distinct genomes $(\mathrm{D}, \mathrm{S}, \mathrm{M}, \mathrm{C}, \mathrm{U}, \mathrm{N}$ and $\mathrm{T})$ were identified in diploids and most of them can be found also in the polyploid Aegilops species. Twelve species in the genus contain $\mathrm{U}$ and/or $\mathrm{M}$ genomes [3] and among them, allopolyploid species evolved from hybridization between diploid Ae. comosa Sm. in Sibth. \& Sm. $(2 \mathrm{n}=2 \mathrm{x}=14, \mathrm{MM})$ and Ae. umbellulata Zhuk. $(2 \mathrm{n}=2 \mathrm{x}=14, \mathrm{UU})$. Allotetraploid Ae. biuncialis Vis. $\left(2 \mathrm{n}=4 \mathrm{x}=28, \mathrm{U}^{\mathrm{b}} \mathrm{U}^{\mathrm{b}} \mathrm{M}^{\mathrm{b}} \mathrm{M}^{\mathrm{b}}\right)$ and $A e$. geniculata Roth. $\left(2 \mathrm{n}=4 \mathrm{x}=28, \mathrm{U}^{\mathrm{g}} \mathrm{U}^{\mathrm{g}} \mathrm{M}^{\mathrm{g}} \mathrm{M}^{\mathrm{g}}\right)$ exhibit the largest ecological adaptation ability [3]. These species are valuable sources of useful genes for wheat breeding. Among others, they were used as source of resistance genes to leaf rust, stripe rust, and powdery mildew (Lr9, Lr57, Sr34, $1 r 8, \operatorname{Tr} 40, \operatorname{Pm} 29)$, which were transferred successfully into wheat [4,5]. Moreover, some genotypes of these species were found tolerant to increased salt levels, drought, frost and heat stresses $[6,7,8]$. Wild relatives of wheat were also studied as potential recipient species for pollenmediated transgene escape from wheat $[9,10,11]$.

Despite extensive research efforts, introgression of favourable agronomic traits from Aegilops species to cultivated wheat remains difficult and the production and identification of wheat-Aegilops introgression lines by molecular cytogenetic methods is time consuming. Only a small number of $\mathrm{U}$ and $\mathrm{M}$ genome-specific molecular markers are available $[12,13,14]$, a fact that limits marker-assisted selection of wheat-Aegilops introgression lines. The lack of suitable markers slows down development of high density genetic and physical maps, mapping of favourable agronomic traits and map-based positional cloning of genes.

Development of molecular markers from particular chromosomes and chromosome arms is an elegant way to saturate genetic maps at regions of interest. Various approaches were employed in several crops to achieve this, such as the use of short-insert 
chromosome-specific DNA libraries enriched for microsatellites $[15,16,17]$ and sequencing ends of clones from chromosomespecific BAC libraries $[18,19]$. These approaches relied on construction of libraries from DNA of chromosomes purified by flow cytometry. However, Wenzl et al. [20] demonstrated that DArT markers can be developed in large numbers directly from a small amount of DNA prepared from flow-sorted chromosomes. The advent of the second generation sequencing technology [21] provides an opportunity to skip DNA library construction and identify sequences suitable for development of DNA markers, including SNPs. For example, Berkman et al. [22] sequenced wheat chromosome arm 7DS to $34 \times$ coverage using Illumina and assembled low copy and genic regions of this chromosome. The assembly represents approximately $40 \%$ of the chromosome arm and all known 7DS genes. Mayer et al. [23,24] sequenced barley chromosome $1 \mathrm{H}$ and arms of chromosomes $2 \mathrm{H}-7 \mathrm{H}$ using 454 to about $2 \times$ coverage and assigned 21,766 barley genes to individual chromosome arms and arranged them in a putative linear order based on conserved synteny with genomes of rice, sorghum and Brachypodium.

To date, flow-cytometric analysis of mitotic chromosomes has been reported in seventeen plant species, including cultivated cereals with economic importance such as hexaploid and tetraploid wheat, barley, rye, oats, rice and maize $[25,26]$. Nevertheless, flow cytometric chromosome analysis and sorting has not been reported in any of their wild relatives. As the technology would greatly aid in transferring genes from wild relatives to cultivated wheat, we set out to explore a possibility of isolating by flow sorting individual chromosomes from $A e$. umbellulata and Ae. comosa and from their natural allotetraploid hybrids Ae. biuncialis and Ae. geniculata. Chromosomes were sorted from individual peaks of flow karyotypes and were identified by FISH with a set of repetitive DNA probes. DNA amplified from isolated chromosomes was used as a template for PCR with SSR markers with the aim to identify their genomic location in the Aegilops species. The results of the present work provide an important step towards analyzing molecular organization of chromosomes in wild relatives of wheat and in developing tools to support alien gene transfer in wheat improvement programmes.

\section{Materials and Methods}

\section{Plant material}

Aegilops umbellulata accession MvGB470, Ae. comosa accession MvGB1039, Ae. biuncialis accession MvGB382 and Ae. geniculata accession AE1311/00 were used for flow cytometric chromosome analysis and sorting, for in situ hybridization experiments and for microsatellite marker analysis. A partial set of wheat-Ae. geniculata disomic addition lines produced by Friebe et al. [27] comprising additions $1 \mathrm{U}^{\mathrm{g}}, 2 \mathrm{U}^{\mathrm{g}}, 3 \mathrm{U}^{\mathrm{g}}, 4 \mathrm{U}^{\mathrm{g}}, 5 \mathrm{U}^{\mathrm{g}}, 6 \mathrm{U}^{\mathrm{g}}, 7 \mathrm{U}^{\mathrm{g}}, 1 \mathrm{M}^{\mathrm{g}}, 2 \mathrm{M}^{\mathrm{g}}, 3 \mathrm{M}^{\mathrm{g}}$, $5 \mathrm{M}^{\mathrm{g}}, 6 \mathrm{M}^{\mathrm{g}}, 7 \mathrm{M}^{\mathrm{g}}$, as well as wheat cv. Chinese Spring and $A$. geniculata accession TA2899 (parents of the addition lines) were used to ascertain chromosomal location of wheat SSR markers in the $\mathrm{U}^{\mathrm{g}}$ and $\mathrm{M}^{\mathrm{g}}$ genomes.

\section{Preparation of liquid suspensions of chromosomes}

Suspensions of intact mitotic chromosomes were prepared from synchronized root-tips of young seedlings. Cell cycle synchrony was induced after a treatment with hydroxyurea and cycling cells were accumulated in metaphase using amiprohos-methyl [28]. Suspensions of intact chromosomes were prepared according to Vrána et al. [29]. Briefly, 50 roots were cut $1 \mathrm{~cm}$ from the root tip, fixed in $2 \%(\mathrm{v} / \mathrm{v})$ formaldehyde in Tris buffer at $5^{\circ} \mathrm{C}$ for $20 \mathrm{~min}$. After washing in Tris buffer, the meristem tips were excised and transferred to a tube containing $1 \mathrm{ml}$ of LB01 buffer [30] at pH 9. Metaphase chromosomes were released after homogenization with a Polytron PT1300 homogenizer (Kinematica AG, Littau, Switzerland) at 20,000 rpm for $13 \mathrm{sec}$. The crude suspension was passed through a $50-\mu \mathrm{m}$ pore size nylon mesh to remove large cellular debris and stored on ice until analyzed on the same day.

\section{Flow cytometric chromosome analysis and sorting}

The samples were analyzed using a FACSVantage SE flow cytometer (Becton Dickinson, San José, USA) equipped with argon ion laser set to multiline UV and $300 \mathrm{~mW}$ output power. Chromosome suspensions were stained with DAPI (4',6-diamidino-2-phenylindole) at a final concentration of $2 \mu \mathrm{g} / \mathrm{ml}$ and analyzed at rates of 200-400 particles per second. DAPI fluorescence was acquired through a 424/44 band-pass filter. Approximately 30 thousand chromosomes were analyzed from each sample and the results were displayed as histograms of relative fluorescence intensity (flow karyotypes). In order to verify chromosome content of individual peaks on flow karyotypes, one thousand chromosomes were sorted from each peak at rates of approximately 5-10 per second onto a microscope slide into $15 \mu \mathrm{l}$ drop of PRINS buffer supplemented with $5 \%$ sucrose [31], airdried and used for FISH with probes for DNA repeats that give chromosome-specific fluorescent labeling patterns (see below).

\section{Preparation of mitotic metaphase spreads}

In order to check karyotypes in all Aegilops accessions used for flow cytometric analysis, FISH was carried out on mitotic metaphase spreads prepared from root tips as described by Jiang et al. [32].

\section{Fluorescence in situ hybridization}

Genomic DNA was extracted from fresh leaves of Ae. tauschii (D genome) and Secale cereale (R genome) according to Sharp et al. [33]. Repetitive DNA sequences pSc119.2 and Afa family were amplified from genomic DNA of $S$. cereale and Ae. tauschii, respectively, and labelled with biotin-16-dUTP (Roche, Mannheim, Germany) and digoxigenin-11-dUTP (Roche) using PCR as described by Contento et al. [34] and Nagaki et al. [35], respectively. The 18S-5.8S-26S rDNA clone pTa71 [36] was labelled with $50 \%$ biotin-11-dUTP and 50\% digoxigenin-11dUTP by nick-translation using standard kits (Roche) following the manufacturer's instructions. Digoxigenin and biotin were detected using anti-digoxigenin-rhodamine Fab fragments (Roche) and streptavidin-FITC (Roche), respectively.

Pretreatment and stringency washes [37] were applied only for the slides containing root tip metaphase cells. These steps were omitted in experiments with flow-sorted chromosomes. Hybridization mix (30 $\mu \mathrm{l}$ per slide), containing $50 \%$ formamide, $2 \times \mathrm{SSC}$, $10 \%$ dextran sulphate, $20 \mathrm{ng}$ of pTa71, and $70 \mathrm{ng}$ each of the pSc119.2 and Afa family probes in the presence of Salmon sperm DNA, was denatured at $80^{\circ} \mathrm{C}$ for $10 \mathrm{~min}$ and stored on ice for 5 min. The chromosome DNA was denatured in the presence of the hybridization mix at $75^{\circ} \mathrm{C}$ for 6 min and allowed to hybridize overnight at $37^{\circ} \mathrm{C}$. For the detection of the hybridization signals, $10 \mu \mathrm{g} / \mathrm{ml}$ each of streptavidin-FITC and anti-digoxigenin-Rhodamin were used. Finally, the slides were counterstained with $2 \mu \mathrm{g} / \mathrm{ml}$ DAPI and examined with Zeiss Axioskop-2 fluorescence microscope using a Plan Neofluar oil objective $63 \times$, N.A. 1.25 (Zeiss, Oberkochen, Germany) equipped with filter sets appropriate for DAPI, FITG and Rhodamin. Images were acquired with a Spot CCD camera (Diagnostic Instruments, Sterling Heights, USA) and compiled with Image Pro Plus software (Media Cybernetics, Silver Spring, USA). 


\section{Amplification of chromosomal DNA}

Chromosomes were sorted from each peak on flow karyotype in batches of 25-50,000 (equivalent to 20-40 ng DNA) into PGR tubes with $40 \mu \mathrm{l}$ sterile deionized water. The chromosomes were treated with proteinase and their DNA was then amplified by multiple displacement amplification (MDA) using Illustra GenomiPhi V2 DNA Amplification Kit (GE Healthcare, Chalfont St. Giles, United Kingdom) as described by Šimková et al. [38]. The amplified DNA was used as a template for PCR with microsatellite markers.

\section{Microsatellite analysis}

Twenty-four microsatellite (SSR) markers derived from bread wheat and wheat A- and D-genome diploid donors were used in this study (Table 1). Genomic DNA was isolated from T. aestivum cv. Chinese Spring, Ae. umbellulata, Ae. comosa, Ae. biuncialis, Ae. geniculata, and wheat-Ae. geniculata addition lines using Quick Gene-Mini80 kit (FujiFilm, Tokyo, Japan) according to the manufacturer's instructions. PCR was carried out in Eppendorf Mastercycler (Eppendorf,
Hamburg, Germany) with reaction profiles optimised for each primer pair $[39,40]$. PCR reactions were performed in $25 \mu \mathrm{l}$ reaction volume and the reaction mix consisted of $2 \times$ GoTaq Green Master Mix (Promega, Madison, USA) and $0.2 \mu \mathrm{M}$ primers. $25 \mathrm{ng}$ genomic DNA or $2 \mathrm{ng}$ of MDA-amplified DNA of flowsorted chromosomes were used as a template. PCR products were separated on $2.5 \%$ agarose gel along with the O'RangeRuler ${ }^{\text {TM }}$ 50 bp DNA size marker (Fermentas, Vilnius, Lithuania). Electrophoretic patterns were documented and analysed using GeneGenius gel documentation system (Syngene, Cambridge, UK).

\section{Results}

\section{Chromosome analysis using flow cytometry (flow karyotyping)}

The analysis of DAPI-stained chromosome suspensions prepared from diploid and tetraploid Aegilops species resulted in flow karyotypes with four peaks (Figure 1). However, there were differences between the species in the degree of resolution of

Table 1. Wheat microsatellite (SSR) markers used in the present study, their assignment to peaks I-IV on flow karyotypes of Ae. umbellulata, Ae. comosa, Ae. biuncialis and Ae. geniculata and their positions on Aegilops chromosomes.

\begin{tabular}{|c|c|c|c|c|c|c|c|}
\hline \multirow[t]{2}{*}{ Marker } & \multirow[t]{2}{*}{$\begin{array}{l}\text { Chromosome } \\
\text { position in wheat }\end{array}$} & \multicolumn{4}{|c|}{ Assignment to peaks on flow karyotypes } & \multicolumn{2}{|c|}{$\begin{array}{l}\text { Chromosome position in } \\
\text { Aegilops }\end{array}$} \\
\hline & & Ae. umbellulata & Ae. comosa & Ae. biuncialis & Ae. geniculata & $\begin{array}{l}\text { Isolated } \\
\text { chromosomes \# }\end{array}$ & $\begin{array}{l}\text { Aegilops } \\
\text { additions \#\# }\end{array}$ \\
\hline Xgdm33 & $1 \mathrm{ABD}$ & 1 & - & 1 & 1 & $1 \mathrm{U}, 1 \mathrm{U}^{\mathrm{b}}$ & na \\
\hline Xgwm164 & $1 \mathrm{~A}$ & - & - & - & - & - & - \\
\hline Xgwm232 & $1 \mathrm{D}, 5 \mathrm{D}$ & - & - & - & - & - & - \\
\hline Xgwm357 & $1 \mathrm{~A}$ & - & - & - & - & - & - \\
\hline Xcfd59* & $1 \mathrm{ABD} / 6 \mathrm{~B}$ & I & 1 & $|+| I \mid$ & I+II & $1 \mathrm{U}, 1 \mathrm{U}^{\mathrm{b}}$ & $1 U^{g}$ \\
\hline Xcfd15 & $1 A D$ & - & - & - & - & - & - \\
\hline Xgwm391* & $3 \mathrm{~A}$ & III & - & - & - & $3 U$ & $3 U^{g}$ \\
\hline Xgwm383 & $3 \mathrm{DB}$ & - & - & - & - & - & - \\
\hline Xgwm114* & $3 A B D$ & - & - & - & II & - & $3 U^{g}$ \\
\hline Xcfa2134* & $3 A B$ & III & - & II & II & $3 U$ & $3 U^{g}$ \\
\hline Xgwm $165^{*}$ & $4 A B D$ & IV & 1 & $\|+I I I$ & III+IV & - & $4 U^{g}$ \\
\hline Xgdm $34^{*}$ & $4 \mathrm{D}$ & IV & - & III & III & - & $5 U^{g}$ \\
\hline Xwmc48* & $4 A B D$ & II & - & II & - & $6 U$ & na \\
\hline Xgwm160 & $4 \mathrm{~A}$ & - & - & - & - & - & - \\
\hline Xgwm205* & $5 A D$ & IV & III & III & III & - & $5 U^{g}$ \\
\hline Xgwm186 & $6 \mathrm{~A}$ & - & - & - & - & - & - \\
\hline Xgwm169 & $6 \mathrm{~A}$ & - & - & - & - & - & - \\
\hline Xgdm142 & 7D & - & - & - & - & - & - \\
\hline Xgwm44* & 7D & - & III & II & - & - & na \\
\hline Xcfa2040* & $7 A B D$ & II & IV & II & II+IV & $6 U$ & na \\
\hline Xgwm635* & $7 A D$ & - & IV & III & - & - & na \\
\hline Xgwm63 & 7D & - & - & - & - & - & - \\
\hline Xbarc111 & $7 D$ & - & - & - & - & - & - \\
\hline Xbarc126* & $7 D$ & - & III+IV & - & IV & - & na \\
\hline \multicolumn{8}{|c|}{$\begin{array}{l}\text { *: Markers tested in wheat-Ae. geniculata addition lines. } \\
\text { na: Ae. geniculata specific fragment was not amplified. } \\
\text { \#: Location of the marker on Aegilops chromosomes was determined according to the presence in the peaks on flow karyotypes. } \\
\text { \#\#: Location of the marker on Aegilops chromosomes was determined according to the presence in the wheat-Ae. geniculata addition lines. } \\
\text { Xgwm: Röder et al. [39]; Xgdm: Pestsova et al. [60]; Xcfa: Sourdille et al. [65]; Xwmc: Wheat Microsatellite Consortium (http://wheat.pw.usda.gov); Xbarc: The US Whea } \\
\text { and Barley Scab Initiative (http://www.scabusa.org). } \\
\text { doi:10.1371/journal.pone.0027708.t001 }\end{array}$} \\
\hline
\end{tabular}



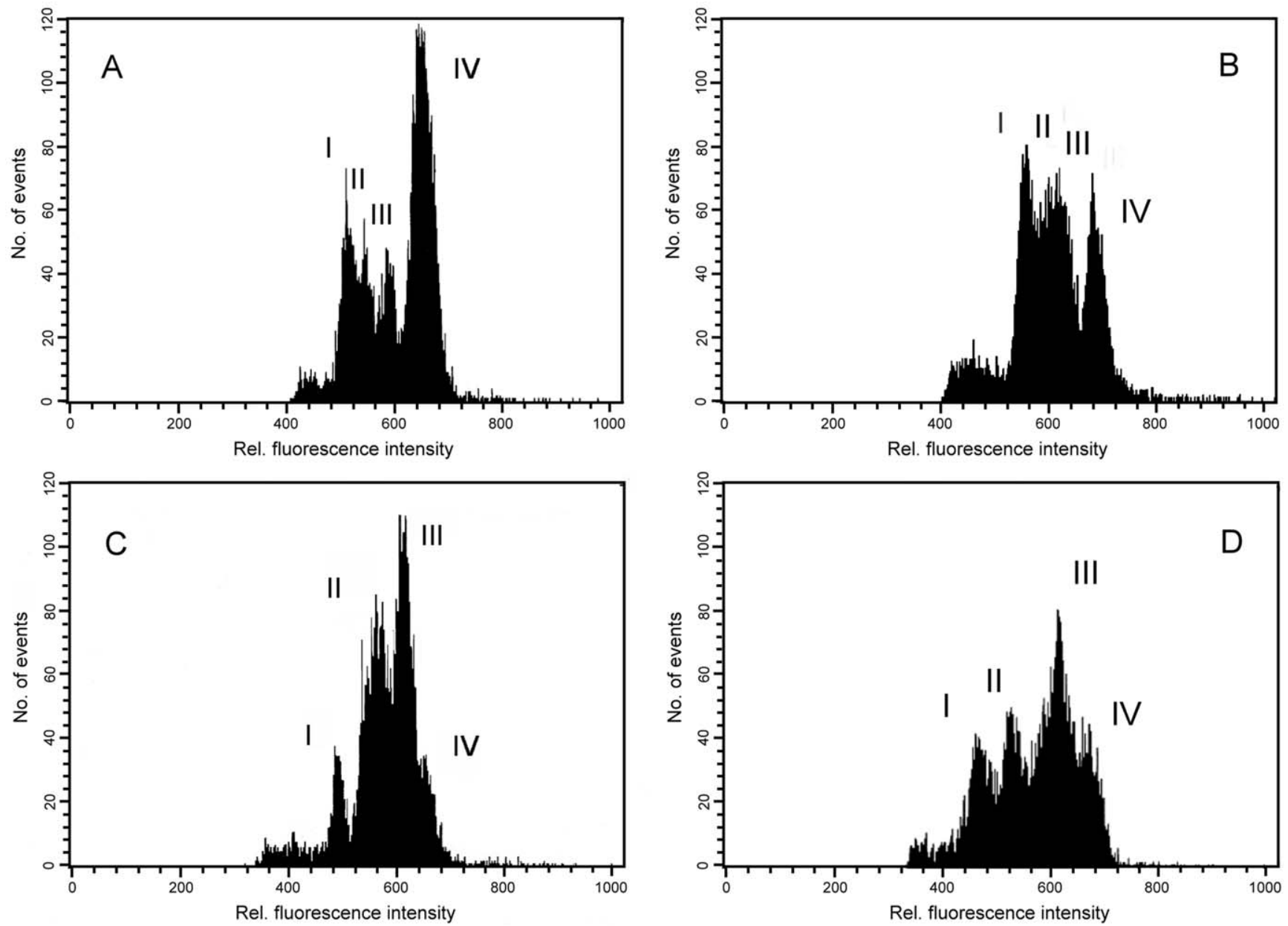

Figure 1. Flow cytometric chromosome analysis. Histograms of relative fluorescence intensity (flow karyotypes) were obtained after the analysis of DAPI-stained chromosome suspensions prepared from Ae. umbellulata MvGB470 ( $n=2 x=14$, UU) (A); and Ae. comosa MvGB1039 $(2 n=2 x=14, M M)(B) ;$ and from their natural hybrids Ae. biuncialis MvGB382 $\left(2 n=4 x=28, U^{b} U^{b} M^{b} M^{b}\right)(C)$; and Ae. geniculata $A E 1311 / 00(2 n=4 x=28$, $\left.\mathrm{U}^{9} U^{9} M^{9} M^{9}\right)(D)$.

doi:10.1371/journal.pone.0027708.g001

individual peaks and their position on flow karyotype. While the first three peaks (I-III) on flow karyotype of Ae. umbellulata were well discriminated from the composite peak IV (Figure 1A), in Ae. comosa peaks II and III could be resolved only partially (Figure 1B). Moreover, chromosome peaks on flow karyotype of Ae. comosa were observed at higher fluorescence intensity channels (560-680) as compared to those of Ae. umbellulata (channels 480-640). Flow karyotype of allotetraploid Ae. biuncialis comprised one single chromosome peak and three composite peaks (Figure 1G). They were observed at similar positions (channels 480-640) as those of $A e$. umbellulata. Flow karyotype of allotetraploid Ae. geniculata comprised four peaks which were located at channels 460-680 (Figure 1D). The range of peak positions in this species corresponded to overlapping ranges of peak positions of Ae. umbellulata (channels 480640) and Ae. comosa (channels 560-680).

\section{FISH on mitotic metaphase spreads}

We could not exclude a possibility that karyotypes of Aegilops accessions used in the present work differed from the reference karyotypes $[41,37,42]$. Thus, FISH with repetitive DNA probes (Afa family, pSc119.2, pTa71) was made on mitotic metaphase spreads to obtain chromosome-specific hybridization patterns for Ae. umbellulata, Ae. comosa, Ae. biuncialis and Ae. geniculata (Figure 2).
Only minor differences were observed in FISH hybridization patterns between the Aegilops genotypes used in this study and those used previously [42]. For example, the lack of a terminal pSc1 19.2 band was observed at the long arm of $2 \mathrm{M}^{\mathrm{b}}$ and the short arm of $7 \mathrm{M}^{\mathrm{b}}$ in the Ae. biuncialis MvGB382. Chromosome $4 \mathrm{M}^{\mathrm{g}}$ of Ae. geniculata AE1311/00 showed no hybridization signals, while a terminal pSc119.2 signal was detected on the $5 \mathrm{U}^{\mathrm{g}} \mathrm{L}$. However, identification of chromosomes was not hindered by these differences and all chromosomes of diploid and tetraploid Aegilops species could be distinguished according to their fluorescence labelling patterns.

\section{Description of flow karyotypes}

Chromosome content of individual peaks was determined after FISH on flow-sorted chromosomes with probes for Afa family, pSc119.2 and pTa71 repeats (Figure 3, Table 2). Strong fluorescent signals of FISH probes were similar to those observed on mitotic metaphase spreads and allowed unambiguous identification of chromosomes in particular peaks of flow karyotypes. In Ae. umbellulata, peak I corresponded to chromosome $1 \mathrm{U}$, which could be sorted at purities $>95 \%$ (Table 2) Peaks II and III predominantly contained chromosomes $6 \mathrm{U}$ and $3 \mathrm{U}$, respectively, which were sorted at purities $74.1 \%$ and $86.4 \%$, respectively. 


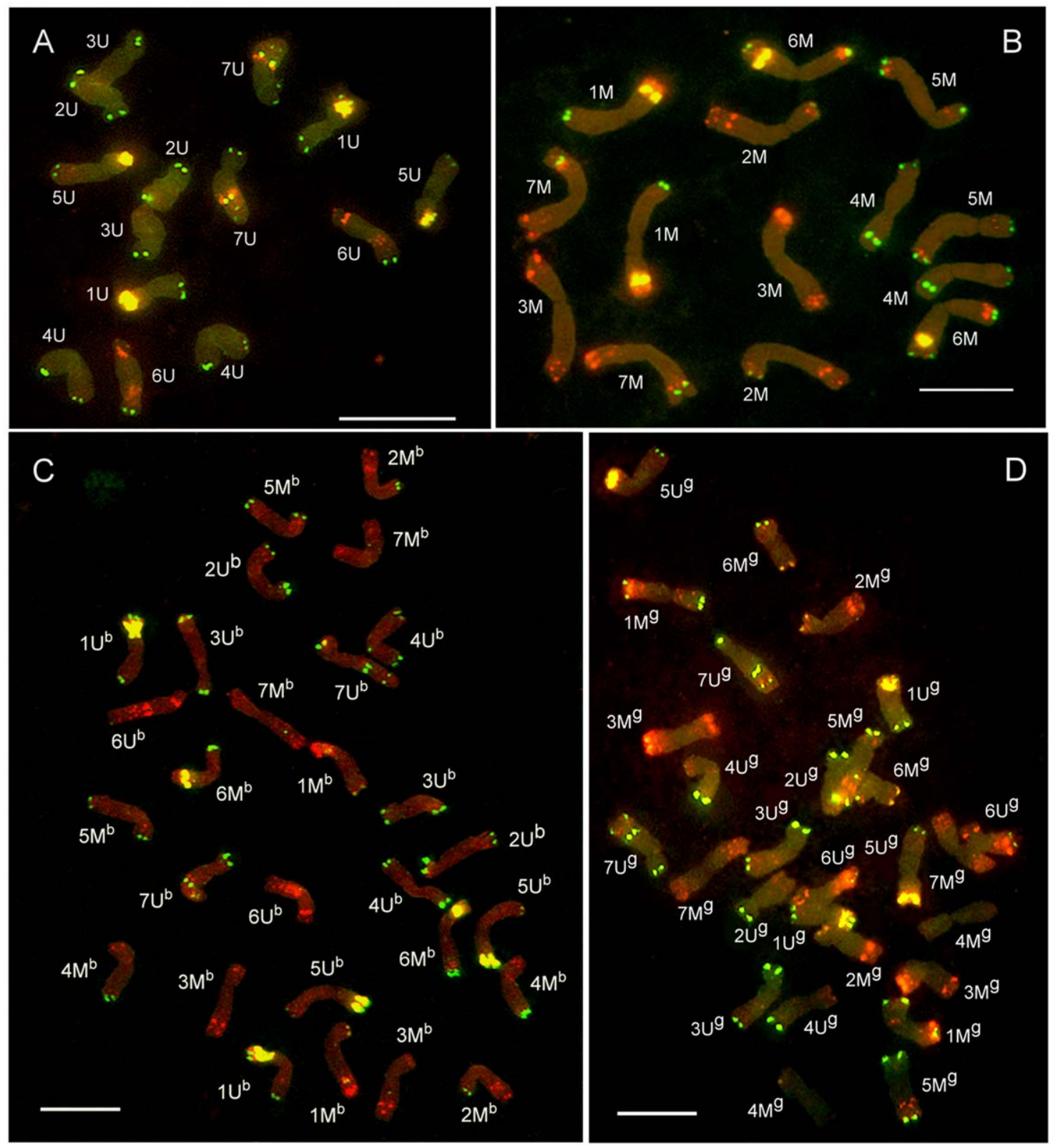

Figure 2. Distribution of DNA repeats on mitotic chromosomes. Fluorescence in situ hybridization (FISH) was done with probes for repetitive DNA: pSc119.2 (green), Afa family (red) and pTa71 (yellow) on mitotic metaphase spreads of Ae. umbellulata MvGB470 (A); Ae. comosa MvGB1039 (B); Ae. biuncialis MvGB382 (C); and Ae. geniculata AE1311/00 (D). Scale bars $=10 \mu \mathrm{m}$. doi:10.1371/journal.pone.0027708.g002

As expected, the composite peak IV represented chromosomes $2 \mathrm{U}, 4 \mathrm{U}, 5 \mathrm{U}$ and $7 \mathrm{U}$. In $A e$. comosa, peak I contained chromosomes $1 \mathrm{M}$ and $4 \mathrm{M}$ as the main fractions, while the peaks II and III, which were closely spaced, represented mainly chromosomes $6 \mathrm{M}$ and $5 \mathrm{M}$, respectively. Chromosome $2 \mathrm{M}$ was present in fractions sorted from both composite peaks (II and III). Finally, peak IV contained chromosomes $3 \mathrm{M}$ and $7 \mathrm{M}$.

FISH analysis showed significant differences between flow karyotypes of allotetraploids $A e$. biuncialis $\left(\mathrm{U}^{\mathrm{b}} \mathrm{U}^{\mathrm{b}} \mathrm{M}^{\mathrm{b}} \mathrm{M}^{\mathrm{b}}\right)$ and $A e$. geniculata $\left(\mathrm{U}^{g} \mathrm{U}^{g} \mathrm{M}^{g} \mathrm{M}^{\mathrm{g}}\right.$ ) (Figure 3, Table 2). In Ae. biuncialis, chromosome $1 \mathrm{U}^{\mathrm{b}}$ could be sorted at high purities $(>95 \%)$ as it was represented by well separated peak I (Figure 1C, Figure 3, Table 2). Peak II contained chromosomes $2 \mathrm{M}^{\mathrm{b}}, 3 \mathrm{M}^{\mathrm{b}}, 4 \mathrm{M}^{\mathrm{b}}, 6 \mathrm{M}^{\mathrm{b}}$, $3 \mathrm{U}^{\mathrm{b}}$, and $6 \mathrm{U}^{\mathrm{b}}$, while peak III represented chromosomes $1 \mathrm{M}^{\mathrm{b}}, 3$
$\mathrm{M}^{\mathrm{b}}, 5 \mathrm{M}^{\mathrm{b}}, 2 \mathrm{U}^{\mathrm{b}}, 4 \mathrm{U}^{\mathrm{b}}, 5 \mathrm{U}^{\mathrm{b}}$ and $7 \mathrm{U}^{\mathrm{b}}$. Chromosome $3 \mathrm{M}^{\mathrm{b}}$ was observed in these two peaks with similar frequency. Peak IV represented largely chromosome $7 \mathrm{M}^{\mathrm{b}}$, which could be sorted at purities exceeding $80 \%$. On the other hand, peak I of Ae. geniculata contained chromosomes $1 \mathrm{U}^{\mathrm{g}}$ and $6 \mathrm{M}^{\mathrm{g}}$, while the peak II represented chromosomes $3 \mathrm{U}^{g}, 4 \mathrm{U}^{g}$ and $6 \mathrm{U}^{g}$. Chromosomes $2 \mathrm{M}^{\mathrm{g}}, 4 \mathrm{M}^{\mathrm{g}}, 5 \mathrm{M}^{\mathrm{g}}, 2 \mathrm{U}^{\mathrm{g}}, 5 \mathrm{U}^{\mathrm{g}}$ and $7 \mathrm{U}^{\mathrm{g}}$ were found in peak III, while the largest chromosomes $1 \mathrm{M}^{\mathrm{g}}, 3 \mathrm{M}^{\mathrm{g}}$ and $7 \mathrm{M}^{\mathrm{g}}$ were represented by peak IV (Figure 1D, Figure 3, Table 2).

Assignment of microsatellite markers to peaks on flow karyotypes

A set of wheat SSR markers was mapped to Aegilops chromosomes using genomic DNA and subgenomic DNA from 


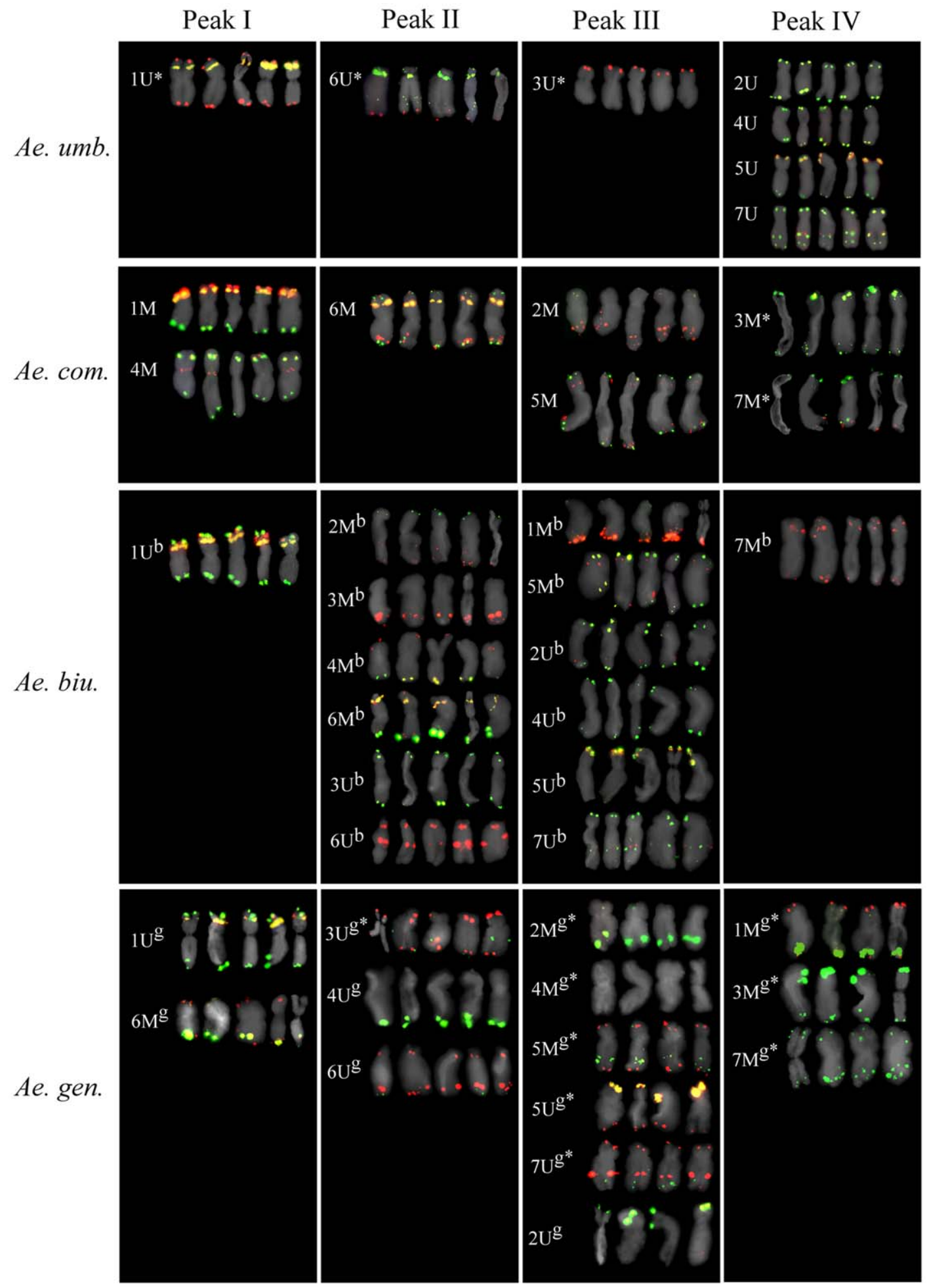

Figure 3. Flow-sorted chromosomes. Mitotic metaphase chromosomes were sorted from individual peaks of flow karyotypes of Ae. umbellulata, Ae. comosa, Ae. biuncialis and Ae. geniculata. Sorted chromosomes were identified after FISH with probes for repetitive DNA: pSc119.2 (green), Afa family (red) and pTa71 (yellow) (Inverse labelling of probes is marked by asterisk). The chromosomes are shown in peaks where their frequencies were the highest. For each chromosome, four to five representative examples are given. doi:10.1371/journal.pone.0027708.g003

individual peaks of flow karyotypes. Out of twenty-four SSR markers, thirteen showed one or two species-specific PCR products in at least one of the Aegilops species (Figure 4, Table 1). PGR products of these markers were also detected with the expected size in one or more peaks of the given species (Figure 4). For example, marker Xcfa2040, which is specific for wheat chromosome group 7 (7ABD), gave a single PCR product in diploid Ae. umbellulata and Ae. comosa and in allotetraploid Ae. 
Table 2. Assignment of chromosomes to peaks of flow karyotypes of Ae. umbellulata, Ae. comosa and their natural tetraploid hybrids Ae. biuncialis and Ae. geniculata.

\begin{tabular}{|c|c|c|c|c|c|c|}
\hline & Genome & Chromosome & Peak I & Peak II & Peak III & Peak IV \\
\hline \multirow[t]{7}{*}{ Ae. umbellulata } & u & 1 & 98.9 & 25.8 & 4.32 & - \\
\hline & & 2 & - & - & - & 31.3 \\
\hline & & 3 & - & - & 86.4 & - \\
\hline & & 4 & - & - & - & 16.0 \\
\hline & & 5 & - & - & - & 30.6 \\
\hline & & 6 & - & 74.1 & 9.25 & - \\
\hline & & 7 & 1.03 & - & - & 22.0 \\
\hline No. of chromosomes analysed & & & 97 & 120 & 162 & 150 \\
\hline \multirow[t]{7}{*}{ Ae. comosa } & M & 1 & 44.4 & 16.34 & - & - \\
\hline & & 2 & 2.5 & 30.7 & 34.2 & - \\
\hline & & 3 & - & - & - & 52.4 \\
\hline & & 4 & 37.6 & - & - & - \\
\hline & & 5 & 3.4 & 4.28 & 63.8 & - \\
\hline & & 6 & 11.9 & 47.1 & 1.8 & - \\
\hline & & 7 & - & - & - & 47.5 \\
\hline No. of chromosomes analysed & & & 117 & 104 & 108 & 82 \\
\hline \multirow[t]{14}{*}{ Ae. biuncialis } & $U^{b}$ & 1 & 95.94 & 0.46 & - & - \\
\hline & & 2 & 0.37 & 1.38 & 21.35 & 0.625 \\
\hline & & 3 & 1.1 & 21.75 & - & - \\
\hline & & 4 & - & 0.46 & 7.76 & 3.125 \\
\hline & & 5 & - & - & 12.62 & 0.625 \\
\hline & & 6 & 2.58 & 8.79 & - & - \\
\hline & & 7 & - & - & 15.53 & 9.375 \\
\hline & $\mathrm{M}^{\mathrm{b}}$ & 1 & - & - & 10.19 & 1.25 \\
\hline & & 2 & - & 25.0 & - & - \\
\hline & & 3 & - & 13.8 & 13.59 & - \\
\hline & & 4 & - & 19.44 & - & 0.625 \\
\hline & & 5 & - & - & 18.44 & 2.5 \\
\hline & & 6 & - & 8.79 & - & 0.625 \\
\hline & & 7 & - & - & 0.48 & 81.25 \\
\hline No. of chromosomes analysed & & & 271 & 216 & 206 & 160 \\
\hline \multirow[t]{14}{*}{ Ae. geniculata } & $U^{g}$ & 1 & 52.0 & 1.7 & - & - \\
\hline & & 2 & 2.6 & 2.6 & 24.3 & - \\
\hline & & 3 & 1.3 & 27.8 & 2.8 & - \\
\hline & & 4 & 5.3 & 28.7 & - & - \\
\hline & & 5 & - & - & 21.5 & - \\
\hline & & 6 & 0.6 & 37.4 & 1.8 & - \\
\hline & & 7 & - & - & 11.2 & - \\
\hline & $M^{g}$ & 1 & - & 0.8 & - & 28.8 \\
\hline & & 2 & - & - & 12.1 & - \\
\hline & & 3 & - & - & - & 42.3 \\
\hline & & 4 & - & - & 14.0 & 3.8 \\
\hline & & 5 & - & - & 13.0 & - \\
\hline & & 6 & 38.0 & 0.8 & - & - \\
\hline & & 7 & - & - & - & 25.0 \\
\hline No. of chromosomes analysed & & & 150 & 115 & 107 & 104 \\
\hline
\end{tabular}

The numbers represent percentage of particular chromosome type from the whole peak content. doi:10.1371/journal.pone.0027708.t002 


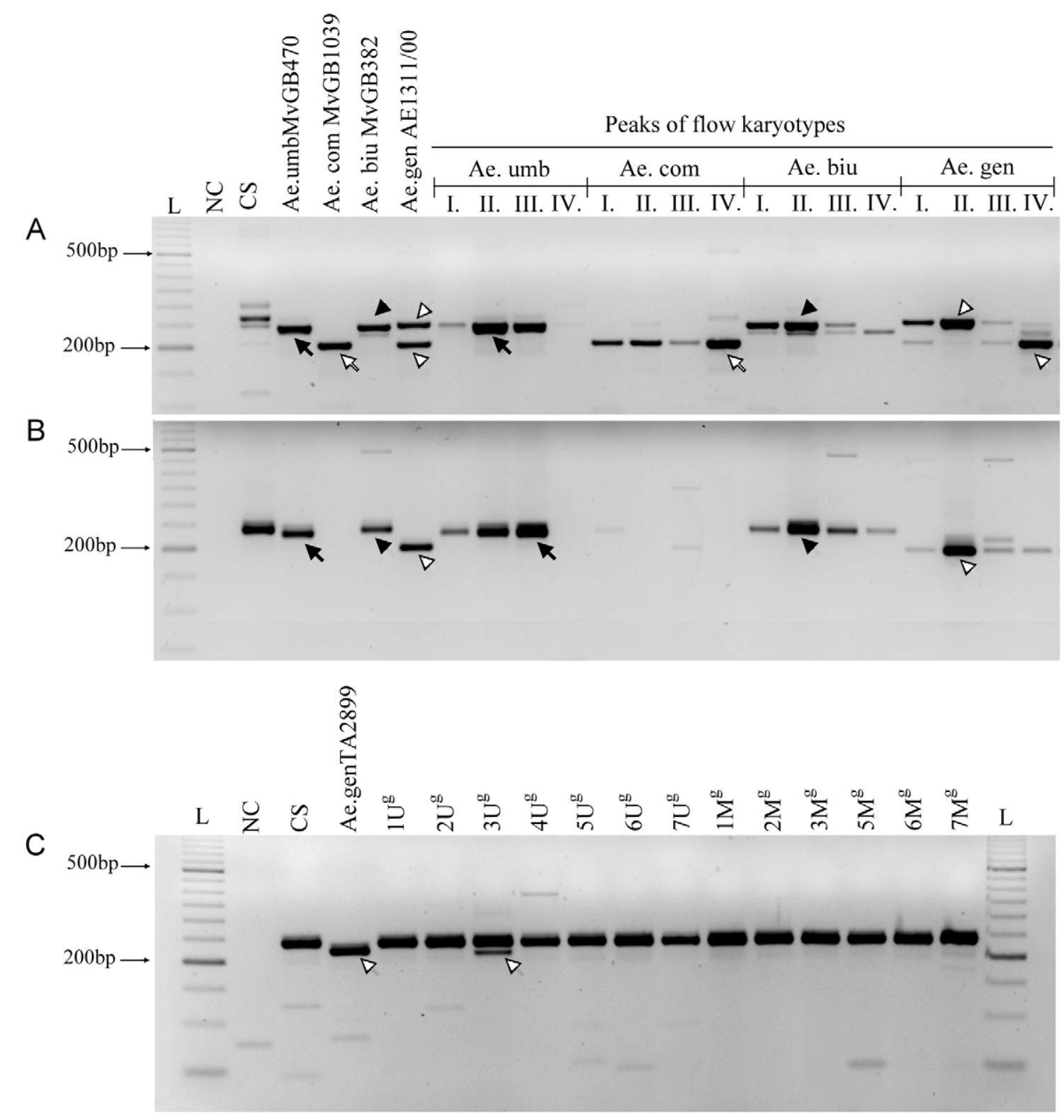

Figure 4. Physical mapping of microsatellite (SSR) markers. PCR products of wheat SSR markers Xcfa2040 (A); and Xcfa2134 (B-C) amplified from wheat cv. Chinese Spring, Ae. umbellulata MvGB470, Ae. comosa MvGB1039, Ae. biuncialis MvGB382 and Ae. geniculata AE1311/00 and from chromosomes of particular peaks of flow karyotypes (I, II, III, IV) (A-B); and from wheat-Ae. geniculata TA2899 addition lines (C). Black arrows, white arrows, black arrowheads and white arrowheads indicate bands specific for Ae. umbellulata, Ae. comosa, Ae. biuncialis and Ae. geniculata, respectively. doi:10.1371/journal.pone.0027708.g004

biuncialis, while two products of different size were observed in allotetraploid Ae. geniculata (Figure 4A). PCR on chromosomes sorted from peaks I, II and III yielded different amounts of the product with size typical of Ae. umbellulata. The strongest PCR product was detected at peak II, which comprises mainly chromosome $6 \mathrm{U}$ (Table 2). The marker gave the strongest PCR product on chromosomes isolated from peak IV of Ae. comosa, which contains chromosomes $3 \mathrm{M}$ and $7 \mathrm{M}$. The strongest product of Xcfa2040 was observed in Ae. biuncialis in the $6 \mathrm{U}^{\mathrm{b}}$ rich peak II, while in Ae. geniculata one of the bands relating to Xcfa2040 was produced predominantly from the $6 \mathrm{U}^{\mathrm{g}}$ rich peak II, whereas the other Xcfa2040 band was produced from peak IV comprising $3 \mathrm{M}^{\mathrm{g}}$ and $7 \mathrm{M}^{\mathrm{g}}$. The positions of the strongest PCR products indicate that the $X_{c f a 2040}$ marker has a locus on $6 \mathrm{U}$ chromosomes of $A e$. umbellulata, Ae. biuncialis and Ae. geniculata and another locus on chromosome 3M and/or 7M in Ae. comosa and Ae. geniculata.

Similar to Xcfa2040, most of the markers gave specific products for more than one peak, which was consistent with the cytological results (Table 2) and could be explained by overlap of the peaks in the flow karyotype. Other explanation might be that SSR markers, which are multilocus markers with high variability, may have different loci on chromosomes represented by different peaks. Therefore, twelve out of the thirteen markers giving PCR products in the Aegilops species were tested in a set of wheat-Ae. geniculata addition lines in order to support their chromosomal assignment based on flow-sorted chromosomes. For example, a single band of the marker Xcfa2134 was found strongest in peaks containing most of the $3 \mathrm{U}$ chromosomes (peak III in Ae. umbellulata and peak II in 
Ae. biuncialis and Ae. geniculata) (Figure 4B). This marker gave Aegilops-specific PCR product only in the $3 \mathrm{U}^{\mathrm{g}}$ addition line (Figure 4C) confirming that this marker has locus only on the $3 \mathrm{U}$ chromosomes. The weaker bands observed in the neighbouring peaks most probably resulted from the overlap of the peaks. Thus, the markers were assigned to peaks showing the most intense PCR products. Table 1 provides a summary of assignments of SSR markers to particular peaks of flow karyotypes and their deduced chromosomal locations.

Because of genetic diversity between Ae. geniculata AE1311/00 used for chromosome sorting and the parental genotype TA2899 of the addition lines, only seven SSR markers gave PCR products on Ae geniculata TA2899 whose chromosomal location could be identified on the wheat-Ae. geniculata addition lines thanks to fragment length polymorphism between $T$. aestivum and $A e$. geniculata (Table 1). The chromosomal positions of markers agreed well with the PCR results of the peak-specific DNA and with the cytological data (Table 2).

In summary, out of the twenty-four SSR markers tested, ten $(41.6 \%)$ could be assigned to individual chromosomes of $A e$. umbellulata (1U: Xgdm33, Xcfd59; 3U: Xgwm391, Xcfa2134; 6U:

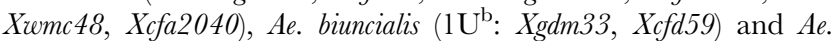

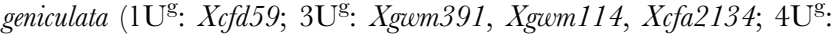

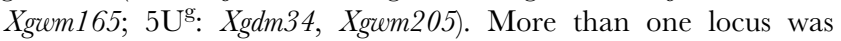
identified for five of them. Possibly more loci exist also for other markers, whose chromosomal location could not be identified unambiguously as they were located in composite peaks, but the Aegilops-specific bands were not detected on the addition lines.

\section{Discussion}

Chromosome analysis and sorting (flow cytogenetics) has been developed in species belonging to tribe Triticeae with high socioeconomic importance such as bread wheat, durum wheat, barley and rye $[29,28,43,44]$. The technology has been a foundation of chromosome genomics [26], an elegant approach to tackle the complex genomes of these crops. Chromosome genomics facilitates the analysis of molecular structure of chromosomes and chromosome arms, high-throughput development of markers, construction of ready-to-sequence physical maps and positional gene cloning. The present work extends the potential of chromosome genomics to wild genetic resources of wheat by establishing flow cytogenetics in two allotetraploid species $A$ e. biuncialis and Ae. geniculata and their diploid progenitors $A e$. umbellulata and Ae. comosa. The protocol for preparation of suspensions of intact chromosomes and chromosome sorting is based on protocols developed for wheat $[28,29]$ and our results in Aegilops species confirm that the original protocol for preparation of suspensions of intact mitotic chromosomes from synchronized root tips [45] can be modified to a range of species.

Flow cytometric analysis of chromosome fluorescence intensity results in a distribution of relative fluorescence intensity (flow karyotype), which is typical for given species and genotype [46]. However, it is rare that every chromosome is represented by a single peak on flow karyotype and it is critical to characterize a flow karyotype and determine chromosome content of each peak. One of the most efficient methods to assign chromosomes to the peaks is FISH [28]. In this work we employed FISH with probes for pSc119.2, Afa family and pTa71 repeats, whose genomic distribution was described previously in Ae. biuncialis and Ae. geniculata as well as in their diploid progenitors Ae. comosa and $A e$. umbellulata $[37,42,47]$.

The fact that chromosome peaks on flow karyotype of Ae. comosa were shifted towards higher fluorescence channels as compared to
Ae. umbellulata suggests that the average size of the $\mathrm{M}$ genome chromosomes, and hence the size of the $\mathrm{M}$ genome is larger than that of the $\mathrm{U}$ genome. These results agree with published $1 \mathrm{C}$ values of Ae. umbellulata (5.05 pg DNA) and Ae. comosa (6.18 pg DNA) [48] and indicate that their genomes underwent different evolutionary changes. Both allotetraploids originated from natural hybridization between Ae. umbellulata and Ae. comosa and have the same genomic constitution (UUMM). The $1 \mathrm{C}$ value of Ae. biuncialis $(11.3 \mathrm{pg})$ is close to the sum of Ae. umbellulata and Ae. comosa genome sizes (11.23 pg DNA) [48], while that of Ae. geniculata (10.29 pg DNA) is significantly less [49]. The evolution of polyploid species is often accompanied by genome rearrangements, including elimination of certain DNA sequences [50,51]. Thus, the $1 \mathrm{C}$ values suggest that $A e$. biuncialis originated more recently from its diploid ancestors than Ae. geniculata. This hypothesis is in good agreement with a genetic diversity study in which Ae. biuncialis showed a much closer relationship to both of its ancestors than Ae. geniculata [13]. These evolutionary changes seem to be manifested at chromosome level as significant differences were observed in chromosome content of peaks on flow karyotypes between two tetraploid Aegilops species, reflecting differences in relative size of homoeologous chromosomes.

In fact, six chromosomes ( $4 \mathrm{U}, 1 \mathrm{M}, 2 \mathrm{M}, 3 \mathrm{M}, 4 \mathrm{M}$ and $6 \mathrm{M}$ ) showed different peak locations in flow karyotypes of Ae. biuncialis and Ae. geniculata. Interestingly, with the exception of chromosome $3 \mathrm{M}$, substantial differences were observed for the same chromosomes in the distribution of satellite sequences and microsatellite motifs $\mathrm{GAA}_{\mathrm{n}}$ and $\mathrm{ACG}_{\mathrm{n}}$ [42]. Moreover, during meiosis in $\mathrm{F}_{1}$ hybrids between wheat-Ae. geniculata addition lines and $A e$. biuncialis, ring bivalents between Ae. geniculata and Ae. biuncialis chromosomes were not observed in those $\mathrm{F}_{1}$ hybrids, which involved chromosome additions $4 \mathrm{U}^{\mathrm{g}}, 1 \mathrm{M}^{\mathrm{g}}, 3 \mathrm{M}^{\mathrm{g}}, 4 \mathrm{M}^{\mathrm{g}}$ and $6 \mathrm{M}^{\mathrm{g}}$ [27]. It may be hypothesized that differences in repetitive DNA and size of these chromosomes may account for blocking the chromosome pairing, which may in turn lead to reproductive isolation of Ae. biuncialis and Ae. geniculata. Different location of chromosomes $4 \mathrm{U}, 1 \mathrm{M}, 2 \mathrm{M}, 3 \mathrm{M}, 4 \mathrm{M}$ and $6 \mathrm{M}$ on flow karyotypes is also in good agreement with the previous results based on STS, RFLP and AFLP markers and karyotype analysis $[37,47,52,53,54]$, indicating that U-genome chromosomes exhibit less interspecific variability than the $\mathrm{M}$ genome partners in the Aegilops species.

Analysis of sorted chromosome fractions by FISH showed that chromosome $1 \mathrm{U}$ could be sorted at more than 95\% purity from $A e$. umbellulata as well as from Ae. biuncialis. However, relatively pure $(74-86 \%)$ fractions of chromosomes $6 \mathrm{U}, 3 \mathrm{U}$ and $7 \mathrm{M}^{\mathrm{b}}$ could also be obtained from these species. Further improvements in the protocol might lead to increased purity of these chromosome fractions in the future. Other chromosomes of the Aegilops species formed composite peaks on flow karyotypes and could be sorted as groups of two to four. A possibility to purify by flow sorting chromosome $1 \mathrm{U}$ from allotetraploid Ae. biuncialis and from its Ugenome diploid progenitor Ae. umbellulata provides opportunities for chromosome survey sequencing using next generation technology. This is a cost effective approach to study molecular composition of individual chromosomes, identify and assemble most of the low-copy sequences and genes on them and order the genes on chromosomes [22,23,55]. The homoeologous group 1 chromosomes of the Triticeae harbour many important genes such as seed storage proteins (HMW glutenins) determining the breadmaking quality in wheat and genes conferring resistance to fungal pathogens (leaf rust, stem rust, yellow rust) and pests (green bug) $[56,57]$. Until now, only one study focused on targeted development of $\mathrm{U}$ and $\mathrm{M}$ genome-specific molecular markers 
using S-SAP (Sequence-Specific Amplified Polymorphism) technology [13]. However, next-generation survey sequencing of $1 \mathrm{U}$ may provide almost unlimited numbers of sequences suitable for development of markers, including SSR, ISBP, and SNP.

The homoeologous group 1 chromosomes $1 \mathrm{H}$ and $1 \mathrm{R}$ can be sorted from barley and rye, respectively [43,44], short arm of $1 \mathrm{R}$ (1RS) can be sorted from wheat-rye ditelosomic addition line [58] and short and long chromosome arms of wheat homoeologous group 1 (1ABD) can be sorted from telosomic lines of wheat [46]. Chromosome $1 \mathrm{H}$ of barley and chromosome arms of homoeologous group 1 of wheat were survey sequenced recently [23,55]. Thus, comparative sequence analysis of chromosome $1 \mathrm{U}$ from Aegilops species with the group 1 chromosomes of wheat, barley and rye is now feasible and may promote the discovery of new wild alleles of important genes for wheat breeding. Such study would also provide detailed insights into the structural changes, which accompanied the evolution of Triticeae genomes and the homoeologous group 1, in particular. Other important use of flow-sorted chromosomes has been the construction of BAC libraries [19], which facilitate targeted development of markers, positional gene cloning and construction of sequence-ready physical maps $[17,18,59]$. To date, BAC libraries have been constructed from 17 chromosomes of wheat (http://lmcc.ieb.cz/dna-libraries). The advance reported in the present work opens a way for constructing BAC libraries from chromosomes $1 \mathrm{U}$ of Aegilops.

Our results obtained after PCR with wheat SSR markers on DNA amplified from flow-sorted Aegilops chromosomes confirm previous observation on suitability of MDA-amplified chromosomal DNA for molecular marker analysis [38]. On the other hand, our results also indicate that the flow-sorted chromosomes can be applied for physical mapping of molecular markers, especially when cytogenetic stocks representing the whole chromosome complements are not available. It must be noted however, that in some cases PCR products of SSR markers specific for the longer chromosomes were also detected in the fraction of shorter chromosomes (peaks I and II), albeit with low intensity. The contamination could be due to fragments of longer chromosomes and their chromatids. Further improvement of the protocol may increase the purity in sorted chromosome fractions.

Wheat SSR markers have been used widely for molecular characterization of different Aegilops species including Ae. biuncialis and Ae. geniculata $[12,14,60,61,62]$. However, the present as well as the earlier observations $[14,63]$ suggest a limited efficiency of wheat SSR markers for characterization of U- and M-genome chromosomes. In this work, Aegilops specific loci were identified for ten wheat SSR markers, four of them (Xgwm114, Xgwm165, $X g d m 34$ and Xgwm205) were mapped earlier on the same Aegilops chromosomes [62]. The markers which identify loci on Aegilops genomes are suitable for marker assisted selection of wheat-Aegilops chromosome addition and translocation lines.

We have found that most of SSR markers were located on the same homoeologous group chromosomes in the Aegilops species as in wheat. However, three markers located in different homoeologous goups. Markers Xgdm34 and Xwmc48 specific for the group 4 chromosomes of wheat were located on Ae. umbellulata chromo- somes $5 \mathrm{U}$ and $6 \mathrm{U}$, respectively, while the marker $X c f a 2040$ specific for the homoeologous group 7 of wheat was identified on chromosome $6 \mathrm{U}$ of Ae. umbellulata. These results are consistent with the study of Zhang et al. [64] who mapped wheat RFLP markers on Ae. umbellulata chromosomes and showed that at least eleven rearrangements differentiate the $\mathrm{D}$ genome of wheat from that of Ae. umbellulata. For example, SSR marker Xcfa2040, which is located on chromosome $6 \mathrm{U}$ according to the present study was physically mapped on long arm of wheat chromosome 7D, bin 7DL3-0.82-1.00 [65]. The RFLP marker XksuD2 was located at similar bin position [66]. The XksuD2 marker was identified later on long arm of chromosome $6 \mathrm{U}$ [64], indicating that the wheat chromosome region represented by markers $X c f a 2040$ and $X k s u \mathrm{D} 2$ changed its chromosomal location in Ae. umbellulata due to the chromosomal rearrangements after the evolutionary divergence of wheat and Ae. umbellulata.

The regions represented by SSR marker Xwmc48 and linked RFLP markers Xpsr 144 and Xpsr139 have also changed their chromosomal location in Ae. umbellulata relative to wheat as these markers were mapped on chromosomes $6 \mathrm{U}$ and 4D (bins 4DS10.57-0.67 and 4DS3-0.67-0.82), respectively [64,65,67]. Similar genome modification was indicated by the group 4-specific SSR marker Xgdm34 and a linked RFLP marker Xpsr164 [65,67], which were mapped to chromosome $5 \mathrm{U}$ in the present study and by Zhang et al. [64].

To conclude, this study marks an important step forward in development of flow cytogenetics for wild genetic resources of wheat. The flow karyotypes of Ae. umbellulata and Ae. comosa and their natural hybrids Ae. biuncialis and Ae. geniculata were characterized and chromosome content of all peaks on karyotypes was determined for the first time. While only chromosome $1 \mathrm{U}$ could be sorted from Ae. umbellulata and Ae. biuncialis with standard karyotype, the results indicate a possibility to sort other chromosome types using accessions with rearranged karyotypes [42]. The ability to purify chromosomes in Aegilops species opens avenues for rapid physical mapping DNA sequences to particular chromosomes using PCR and DNA arrays, construction of chromosome-specific BAC libraries and next generation sequencing to identify low-copy and genic sequences and develop new markers. Wheat SSR markers which were assigned to Aegilops chromosomes in this study could be used in pre-breeding programmes to select chromosome segments carrying agronomically useful genes in wheat-Aegilops translocation lines.

\section{Acknowledgments}

We greatly acknowledge Jarmila Číhalíková, MSc., Peter Navrátil, MSc., and Zdenka Dubská for assistance with chromosome sorting and DNA amplification.

\section{Author Contributions}

Conceived and designed the experiments: IM MK MM-L JD. Performed the experiments: IM MK HŠ AG. Analyzed the data: ÍM MK AC JD. Contributed reagents/materials/analysis tools: IM AC MM-L JD. Wrote the paper: IM HŠ MM-L JD.

\section{References}

1. Sabot F, Guyot R, Wicker T, Chantret N, Laubin B, et al. (2005) Updating of transposable element annotations from large wheat genomic sequences reveals diverse activities and gene associations. Mol Genet Genomics 274: 119-130.

2. Feuillet G, Langridge P, Waugh R (2008) Cereal breeding takes a walk on the wild side. Trends Genet 24: 24-32.

3. Van Slageren MW (1994) Wild wheats: A monograph of Aegilops L. and Amblyopyrum (Jaub and Spach) Eig (Poaceae). Wageningen: Wageningen Agricultural University Papers 94-7: 514.
4. Friebe B, Jiang J, Raupp WJ, McIntosh RA, Gill BS (1996) Characterization of wheat alien translocations conferring resistance to diseases and pests: current status. Euphytica 71: 59-87.

5. Schneider A, Molnár I, Molnár-Láng M (2008) Utilisation of Aegilops (goatgrass) species to widen the genetic diversity of cultivated wheat. Euphytica 163: 1-19.

6. Rekika D, Monneveux P, Havaux M (1997) The in vivo tolerance of photosynthetic membranes to high and low temperatures in cultivated and wild wheats of the Triticum and Aegilops genera. J Plant Physiol 150: 734-738. 
7. Colmer TD, Flowers TJ, Munns R (2006) Use of wild relatives to improve salt tolerance in wheat. J Exp Bot 57: 1059-1078.

8. Molnár I, Gáspár L, Sárvári É, Dulai S, Hoffmann B, et al. (2004) Physiological and morphological responses to water stress in Aegilops biuncialis and Triticum aestivum genotypes with differing tolerance to drought. Funct Plant Biol 31: $1149-1159$.

9. Zaharieva M, Monneveux P (2006) Spontaneous hybridization between bread wheat (Triticum aestivum L.) and its wild relatives in Europe. Crop Sci 46: 512-527.

10. Cifuentes M, Blein M, Benavente E (2006) A cytomolecular approach to assess the potential of gene transfer from a crop (Triticum turgidum L.) to a wild relative (Aegilops geniculata Roth.). Theor Appl Genet 112: 657-664.

11. Cifuentes M, Benavente E (2009) Wheat-alien metaphase I pairing of individual wheat genomes and D genome chromosomes in interspecific hybrids between Triticum aestivum L. and Aegilops geniculata Roth. Theor Appl Genet 119: 805-813.

12. Peil A, Korzun V, Schubert V, Schumann E, Weber WE, et al. (1998) The application of wheat microsatellites to identify disomic Triticum aestivum-Aegilops markgrafii addition lines. Theor Appl Genet 96: 138-146.

13. Nagy ED, Molnár I, Schneider A, Kovács G, Molnár-Láng M (2006) Characterisation of chromosome-specific S-SAP markers and their use to study genetic diversity in Aegilops species. Genome 49: 289-296.

14. Schneider A, Molnár I, Molnár-Láng M (2010) Selection of U and M genomespecific wheat SSR markers using wheat-Aegilops biuncialis and wheat-Ae. geniculata addition lines. Euphytica 175: 357-364.

15. Požárková D, Kobližková A, Román B, Torres AM, Lucretti S, et al. (2002) Development and characterization of microsatellite markers from chromosome 1-specific DNA libraries of Vicia faba. Biol Plantarum 45: 337-345.

16. Román B, Satovic Z, Požárková D, Macas J, Doležel J, et al. (2004) Development of a composite map in Vicia faba, breeding applications and future prospects. Theor Appl Genet 108: 1079-1088.

17. Kofler R, Bartoš J, Gong L, Stift G, Suchánková P, et al. (2008) Development of microsatellite markers specific for the short arm of rye (Secale cereale L.) chromosome 1. Theor Appl Genet 117: 915-926.

18. Bartoš J, Paux E, Kofler R, Havránková M, Kopecký D, et al. (2008) A first survey of the rye (Secale cereale) genome composition through BAC end sequencing of the short arm of chromosome 1R. BMC Plant Biol 8: 95.

19. Šafář J, Šimková H, Kubaláková M, Číhalíková J, Suchánková P, et al. (2010) Development of chromosome-specific BAC resources for genomics of bread wheat. Cytogenet Genome Res 129: 211-223.

20. Wenzl P, Suchánková P, Jason C, Šimková H, Huttner E, et al. (2010) Isolated chromosomes as a new and efficient source of DArT markers for the saturation of genetic maps. Theor Appl Genet 121: 465-474.

21. Edwards D, Batley J (2010) Plant genome sequencing: applications for crop improvement. Plant Biotech J 8: 2-9.

22. Berkman PJ, Skarshewski A, Lorenc MT, Lai K, Duran C, et al. (2011) Sequencing and assembly of low copy and genic regions of isolated Triticum aestivum chromosome arm 7DS. Plant Biotech J 9: 768-775.

23. Maver KFX, Taudien S, Martis M, Šimková H, Suchánková P, et al. (2009) Gene content and virtual gene order of barley chromosome 1H. Plant Physiol 151: 496-505.

24. Mayer KFX, Martis M, Hedley PE, Šimková H, Liu H, et al. (2011) Unlocking the barley genome by chromosomal and comparative genomics. Plant Cell 23: 1249-1263.

25. Doležel J, Kubaláková M, Bartoš J, Macas J (2004) Flow cytogenetics and plant genome mapping. Chromosome Res 12: 77-91.

26. Doležel J, Kubaláková M, Paux E, Bartoš J, Feuillet C (2007) Chromosomebased genomics in the cereals. Chromosome Res 15: 51-66.

27. Friebe B, Tuleen NA, Gill BS (1999) Development and identification of a set of Triticum aestivum - Aegilops geniculata chromosome addition lines. Genome 42: 374-380.

28. Kubaláková M, Kovářová P, Suchánková P, Ćíhalíková J, Bartoš J, et al. (2005) Chromosome sorting in tetraploid wheat and its potential for genome analysis. Genetics 170: 823-829.

29. Vrána J, Kubaláková M, Šimková H, Číhalíková J, Lysák MA, et al. (2000) Flow-sorting of mitotic chromosomes in common wheat (Triticum aestivum L.). Genetics 156: 2033-2041.

30. Doležel J, Binarová P, Lucretti S (1989) Analysis of nuclear DNA content in plant cells by flow cytometry. Biol Plantarum 31: 113-120.

31. Kubaláková M, Macas J, Doležel J (1997) Mapping of repeated DNA sequences in plant chromosomes by PRINS and C-PRINS. Theor Appl Genet 94: 758-763.

32. Jiang JM, Friebe B, Gill BS (1994) Recent advances in alien gene-transfer in wheat. Euphytica 73: 199-212.

33. Sharp PJ, Kreis M, Shewry PR, Gale MD (1988) Location of $\beta$-amylase sequences in wheat and its relatives. Theor Appl Genet 75: 286-290.

34. Contento A, Heslop-Harrison JS, Schwarzacher T (2005) Diversity of a major repetitive DNA sequence in diploid and polyploid Triticeae. Cytogenet Genome Res 109: 34-42.

35. Nagaki K, Tsujimoto H, Isono K, Sasakuma T (1995) Molecular characterization of a tandem repeat, Afa family, and its distribution among Triticeae. Genome 38: 479-486.

36. Gerlach WL, Bedbrook JR (1979) Cloning and characterization of ribosomal RNA genes from wheat and barley. Nucleic Acids Res 7: 1869-1885.
37. Schneider A, Linc G, Molnár I, Molnár-Láng M (2005) Molecular cytogenetic characterization of Aegilops biuncialis and its use for the identification of five derived wheat-Aegilops biuncialis disomic addition lines. Genome 48: 1070-1082.

38. Šimková H, Svensson JT, Condamine P, Hřibová E, Suchánková P, et al. (2008) Coupling amplified DNA from flow-sorted chromosomes to high-density SNP mapping in barley. BMC Genomics 9: 294.

39. Röder MS, Korzun V, Wandehake K, Planschke J, Tixier MH, et al. (1998) A microsatellite map of wheat. Genetics 149: 2007-2023.

40. Somers DJ, Isaac P, Edwards K (2004) A high-density microsatellite consensus map for bread wheat (Triticum aestivum L.). Theor Appl Genet 109: 1105-1114.

41. Badaeva ED, Friebe B, Gill BS (1996) Genome differentiation in Aegilops. 1. Distribution of highly repetitive DNA sequences on chromosomes of diploid species. Genome 39: 293-306.

42. Molnár I, Gifuentes M, Schneider A, Benavente E, Molnár-Láng M (2011) Association between simple sequence repeat-rich chromosome regions and intergenomic translocation breakpoints in natural populations of allopolyploid wild wheats. Ann Bot 107: 65-76.

43. Lysák MA, Číhalíková J, Kubaláková M, Šimková H, Künzel G, et al. (1999) Flow karyotyping and sorting of mitotic chromosomes of barley (Hordeum vulgare L.). Chromosome Res 7: 431-444.

44. Kubaláková M, Valárik M, Bartoš J, Vrána J, Cíhalíková J, et al. (2003) Analysis and sorting of rye (Secale cereale $\mathrm{L}$.) chromosomes using flow cytometry. Genome 46: 893-905.

45. Doležel J, Č́halíková J, Lucretti S (1992) A high-yield procedure for isolation of metaphase chromosomes from root tips of Vicia faba L. Planta 188: 93-98.

46. Kubaláková M, Vrána J, Číhalíková J, Šimková H, Doležel J (2002) Flow karyotyping and chromosome sorting in bread wheat (Triticum aestivum L.). Theor Appl Genet 104: 1362-1372.

47. Badaeva ED, Amosova AV, Samatadze TE, Zoshchuk SA, Shostak NG, et al. (2004) Genome differentiation in Aegilops. 4. Evolution of the U-genome cluster. Plant Syst Evol 246: 45-76.

48. Furuta Y (1970) DNA content per nucleus in Aegilops species. Wheat Information Service 30: 20-22

49. Eilam T, Anikster Y, Millet E, Manisterski J, Feldman M (2008) Nuclear DNA amount and genome downsizing in natural and synthetic allopolyploids of the genera Aegilops and Triticum. Genome 51: 616-627.

50. Wendel JF (2000) Genome evolution in polyploids. Plant Mol Biol 42: 225-249.

51. Feldman M, Levy AA (2005) Allopolyploidy - a shaping force in the evolution of wheat genomes. Cytogenet Genome Res 109: 250-258.

52. Chee PW, Lavin M, Talbert LE (1995) Molecular analysis of evolutionary patterns of U genome wild wheats. Genome 38: 290-297.

53. Resta P, Zhang HB, Dubcovsky J, DvorakJ (1996) The origins of the genomes of Triticum biunciale, T. ovatum, T. neglectum, T. columnare and T. rectum (Poaceae) based on variation in repeated nucleotide sequences. Am J Bot 83: 1556-1565.

54. Monte JV, De Nova PJG, Soler C (2001) AFLP-based analysis to study genetic variability and relationships in the Spanish species of the genus Aegilops. Hereditas 135: 233-238.

55. Wicker T, Mayer KFX, Gundlach H, Martis M, Steuernagel B, et al. (2011) Frequent gene movement and pseudogene evolution is common to the large and complex genomes of wheat, barley, and their relatives. Plant Cell 23: 1706-1718.

56. Payne PI (1987) Genetics of wheat storage proteins and the effect of allelic variation on bread-making quality. Ann Rev Plant Physiol 38: 141-153.

57. Zeller FJ, Fuchs E (1983) Cytology and disease resistance of a 1A/1R and some 1B/1R wheat-rye translocation cultivars. Z Pflanzenzuecht 90: 285-296.

58. Šimková H, Šafář J, Suchánková P, Kovářová P, Bartoš J, et al. (2008) A novel resource for genomics of Triticeae: BAC library specific for the short arm of rye (Secale cereale L.) chromosome 1R (1RS). BMC Genomics 9: 237.

59. Paux E, Sourdille P, Salse J, Saintenac C, Choulet F, et al. (2008) A physical map of the 1-gigabase bread wheat chromosome 3B. Science 322: 101-104.

60. Pestsova E, Korzun V, Goncharov NP, Hammer K, Ganal MW, et al. (2000) Microsatellite analysis of Aegilops tauschii germplasm. Theor Appl Genet 101: $100-106$.

61. Lelley T, Stachel M, Grausgruber H, Vollmann J (2000) Analysis of relationships between Aegilops tauschii and the D genome of wheat utilizing microsatellites. Genome 43: 661-668.

62. Zaharieva M, Suenaga K, William HM, Mujeeb-Kazi A (2003) Microsatellite markers for identification of Aegilops geniculata Roth. M- and U-genome chromosomes in wheat background. Annual Wheat Newsletter 49: 75-78.

63. Zaharieva M, Suenaga K, William HM, Mujeeb-Kazi A (2002) Identification of specific Aegilops geniculata microsatellite markers potentially useful for detecting introgressions into bread wheat. Annual Wheat Newsletter 48: 108-110.

64. Zhang H, Jia J, Gale MD, Devos KM (1998) Relationships between the chromosomes of Aegilops umbellulata and wheat. Theor Appl Genet 96: 69-75.

65. Sourdille P, Singh S, Cadalen T, Brown-Guedira GL, Gay G, et al. (2004) Microsatellite-based deletion bin system for the establishment of geneticphysical map relationships in wheat (Triticum aestivum L.). Funct Integr Genomics 4: $12-25$.

66. Hohmann U, Endo TR, Herrmann RG, Gill BS (1995) Characterization of deletions in common wheat induced by an Aegilops cylindrica 1 chromosome: deletion of multiple chromosome rearrangements. Theor Appl Genet 91: 611-617.

67. Mickelson-Young L, Endo TR, Gill BS (1995) A cytogenetic ladder-map of the wheat homoeologous group-4 chromosomes. Theor Appl Genet 90: 1007-1011. 